\title{
Localization and Quantification of Ultraviolet Radiation Absorbing Compounds in Leaves of Southern Magnolia (Magnolia grandiflora L.)
}

\author{
Vanessa Ferchaud ${ }^{1}$, Yadong $\mathrm{Qi}^{2}$, Veronica Manrique ${ }^{2}$ and Kit Chin $^{2}$ \\ ${ }^{1}$ Southern University and A\&M College, Baton Rouge, Louisiana, United States, ${ }^{2}$ Southern University and \\ A\&M College, United States
}

The stratospheric ozone depletion has been reported for more than four decades. With the uncertainty of ozone layer recovery, there is a need to continue to monitor the plant performance and their responses to the enhanced solar UV-B (280-315 nm) radiation reaching the Earth's surface. UV-B radiation has been shown to be harmful to living organisms, damaging DNA, proteins, lipids, and cell membranes. Plants, which use sunlight for photosynthesis and are unable to avoid exposure to enhanced levels of UV-B radiation, are at risk [1]. Plants are sessile and they must adapt and develop repairing or protective mechanisms to cope with the potential damage caused by UV radiation. Research indicated that one of the important mechanisms responsible for plant tolerance to UV radiation is largely due to its possession of UV absorbing compounds, mainly flavonoids and related phenolic compounds depending on species [2,3].

Forests are accounted for the majority of living biomass on earth; thus, it is important to understand how diverse forest tree species cope with the harmful UV-B radiation as it has a disproportionately large photobiological effect on forest tree species [4]. UV-radiation sensitivity can result in a decrease in leaf area and biomass, and changes to flowering, reproduction, and competition [5]. Evergreen trees can be more beneficial than deciduous trees to urban environment because they offer protection from the sun year around. Southern magnolia (Magnolia grandiflora L.) is a popular landscaping species with large fragrant white flowers and evergreen large leathery leaves that make it one of the most splendid of forest trees [6]. The aim of this study was to localize, visualize, and quantify UV absorbing compounds in Southern magnolia leaves. The goal is to gain biochemical and anatomical insight into the UV tolerance for this species.

Leave samples of Southern magnolia were collected from sun-exposed portions at the terminal end, 10-50 cm of a branch, from five individual Southern magnolia trees in spring (April), summer (August), and fall (November) during a growing season in 2009 in Baton Rouge, LA., USA.

Histochemical Analysis for Localization of UV-absorbing Compounds - The fresh leaves were sectioned into approximately $0.5 \mathrm{~mm} \times 0.5 \mathrm{~mm}$ squares pieces using a sharp razor and embedded on a specimen disc using OTC compound (Jung Tissue Freezing Medium, Leica Microsystems) and placed in the chamber of a cryostat (Leica CM-1850 UV Microtome, Nussloch, Germany) for sectioning at $40 \mu \mathrm{m}$ thickness. Rapid freezing at a low temperature of $-20^{\circ} \mathrm{C}$ was utilized depending on leaf moisture content. One drop of $0.2 \%$ Naturstoffreagenz-A (NA) (from Sigma Aldrich, St. Louis, MO) [0.2\%, w/v, $0.2 \mathrm{~g}$ amino ethyl diphenyl boric acid in $100 \mathrm{ml}$ distilled water stock solution] was added to a slide and then covered with a glass coverslip. Images were taken using the monochrome camera and bright-field color camera with Leica DMI6000 B Inverted Research Automated Robotic Fluorescent Imaging Microscope.

Leaf Extractions of Total UV-absorbing Compound Content for Spectrophotometric Analysis - UV-B absorbing compounds were extracted with acidified methanol (MeOH-H2O-HCL, 79:29:1, v/v) solution. Three leaves of similar size were randomly selected from each of 5 individual tree branches and 1 disc per leaf approximately $6 \mathrm{~mm}$ in diameter was punched from the leaves in the middle of the lamina away from the midrib. The 3 discs were put in a $1.5 \mathrm{ml}$ Fisher brand microcentrifuge tube and liquid nitrogen was added to homogenize the discs using a Teflon pestle. One (1) $\mathrm{ml}$ acidified methanol was added to the microcentrifuge to complete the homogeneous process. This process was carried out repetitiously until all samples were completed for each season. Extracts were stored frozen $\left(-80^{\circ} \mathrm{C}\right)$ for up to 1 week, then clarified by centrifugation at $2500 \mathrm{rpm}$ for $15 \mathrm{~min}$ (Eppendorf model $5415 \mathrm{D}$ Microfuge). Thirty (30) $\mu 1$ of supernatant was diluted in $3 \mathrm{ml}$ of acidified methanol into a $4.5 \mathrm{~mL}$ polystyrn cuvette for analysis. Absorbency spectra of 
the extracts from 190-700 $\mathrm{nm}$ in steps of $\sim 5 \mathrm{~nm}$ was obtained with a UV/Visible Genesys 6 Spectrophotometer (Thermo Electron Corporation).

Results - The NA-stained young Southern magnolia leaves show that the cellular and wall-bound UV absorbing-compounds were rendered highly visible under monochrome camera via green fluorescent protein (GFP) cube (Figs. 1a, 1b). Fig. 1a shows the UV absorbing-compounds were present primarily in upper and lower epidermis, palisade tissues, and trichomes in leaf cross-section. And Fig. 1b shows that the UV absorbing-compounds also present in vascular bundle in addition to epidermal layers and palisade tissues in the petiole. Fig. 1c is the leaf cross-sectional view taken with bright-field camera showing the upper and lower epidermis, leaf hair, palisade and sponge mesophyll, and vascular bundles.

Southern magnolia leaf total UV absorbing compound content was studied in three seasons. The results indicated there was a general increasing trend in total UV absorbing compounds during the leaf developmental stage from spring (April) to summer (August), but no further increase detected in the leaves collected in fall (November) (Fig. 2a). In most species, leaf UV absorbing-compounds are at their highest peaks during the summer when UV radiation is very high. However, in Southern magnolia, the contents of UV-A and UV-B absorbing compounds were not statistically significantly affected by leaf age in the three stages (Fig. 2b). The young leaves in April were as effective as the mature leaves in August and November in absorbing the harmful UV-B and UV-A radiation. This may indicate that Southern magnolia leaves possess the UV tolerance properties in both their young and mature leaves. Furthermore, the UV protection capability may relate to the strategic location of their UV-absorbing compounds presented in the leaves of Southern magnolia

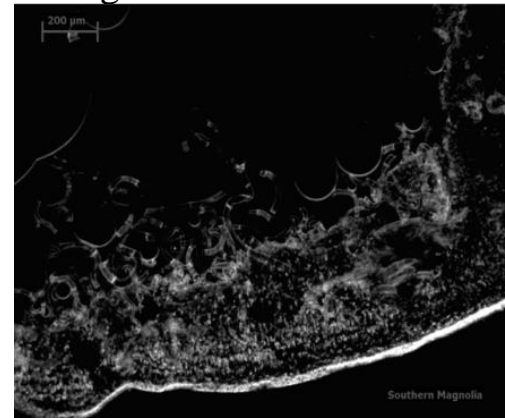

Figure 1a. Southern magnolia young leaf (collected in April) cross-section using GFP-NA-stain with monochrome camera via GFP cube.
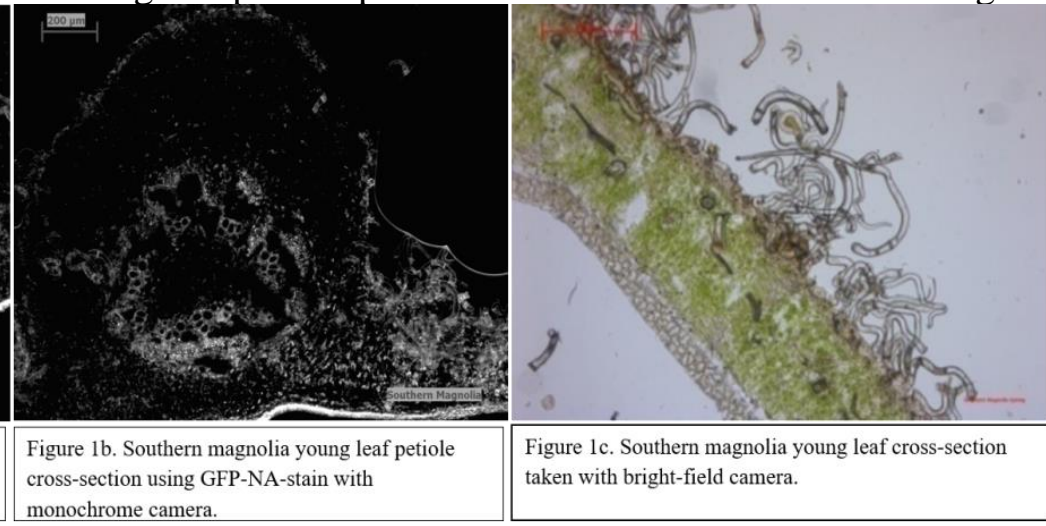

Figure 1 .

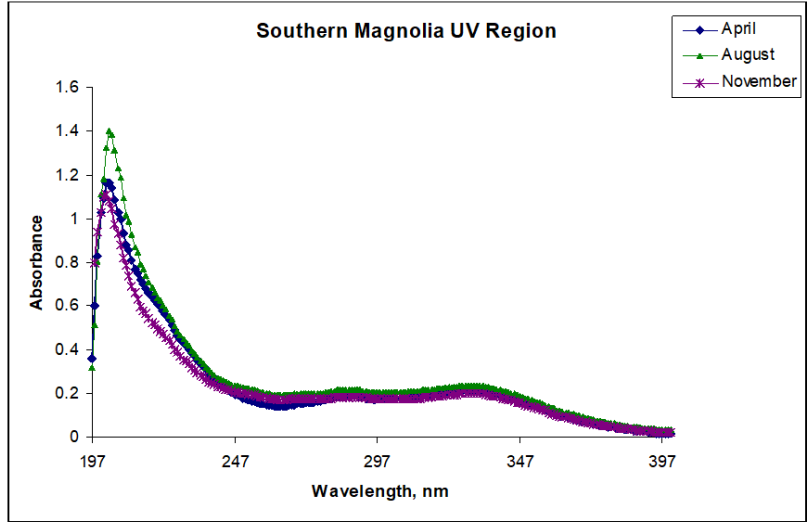

Figure 2a. Southern magnolia total UV-absorbance profile $(200-400 \mathrm{~nm})$ in leaves sampled in three growth stages, April, August, and November, by spectrophotometric analysis.

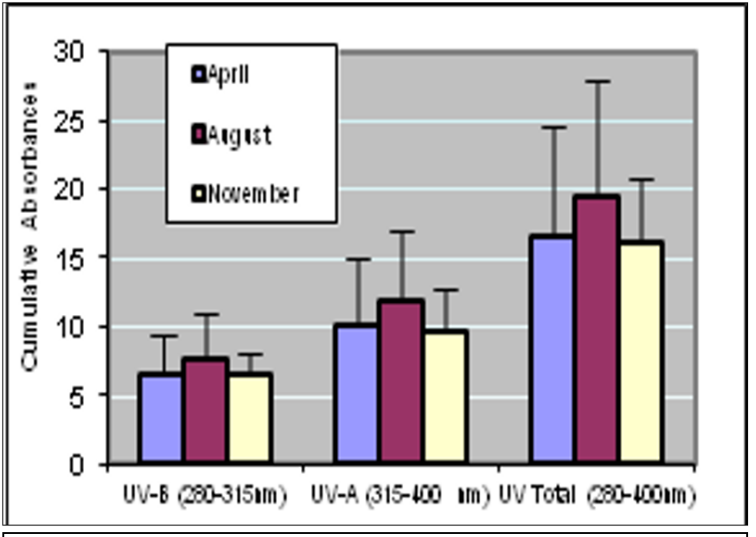

Figure $2 b$. Southern magnolia leaf UV-B, UV-A and UV-total absorbingcompound contents (mean $+1 \mathrm{sd})$ by spectrophotometric analysis.

Figure 2. 


\section{References}

[1] Hollósy, F. 2002. Review: Effects of ultraviolet radiation on plant cells. Micron 33(2):179-97. Doi: 10.1016/s0968-4328(01)00011-7.

[2] Macie, E. (2013). Decrease in Energy Costs from Urban Forests. eXtension. http://www.extension.org/pages/67765/decrease-in-energy-costs-from-urban-forests.

[3] Harris, M.R., Lamb, D. and Erskine, P.D. (2003). An investigation into the possible inhibitory effects of white cypress pine (Callitris glaucophylla) litter on the germination and growth of associated ground cover species. Australian Journal of Botany, 18(51): 93-102.

[4] Singh, S. S., P. Kumar, and A. K. Rai. 2006. Ultraviolet Radiation Stress: Molecular and Physiological Adaptations in Trees, DOI: 10.1007/1-4020-4389-9_6. p91-110. In: Abiotic Stress Tolerance in Plants, Springer.

[5] Caldwell, M. M., A. H. Teramura, M. Tevini, J. F. Bornman, L. Björn, L., and G. Kulandaivelu. (1995). Effects of increased solar ultraviolet radiation on terrestrial plants, Ambio, 24, 166-173.

[6] Outcalt, K.W. (2004). Magnolia grandiflora L. (Southern Magnolia). National Forest Service Silviculture manual.

[7] The authors acknowledge funding from USDA-NIFA Grant 2014-38821-22415. 